\title{
FRACTIONAL POWERS OF HYPONORMAL OPERATORS OF PUTNAM TYPE
}

\author{
TOKA DIAGANA
}

Received 20 January 2005 and in revised form 12 April 2005

We are concerned with fractional powers of the so-called hyponormal operators of Putnam type. Under some suitable assumptions it is shown that if $A, B$ are closed hyponormal linear operators of Putnam type acting on a complex Hilbert space $\mathbb{U}$, then $D\left((\overline{A+B})^{\alpha}\right)=D\left(A^{\alpha}\right) \cap D\left(B^{\alpha}\right)=D\left((\overline{A+B})^{* \alpha}\right)$ for each $\alpha \in(0,1)$. As an application, a large class of the Schrödinger's operator with a complex potential $Q \in L_{\text {loc }}^{1}\left(\mathbb{R}^{d}\right)+L^{\infty}\left(\mathbb{R}^{d}\right)$ is considered.

\section{Introduction}

Let $\mathbb{W}$ be a complex Hilbert space. A linear (possibly not continuous) operator $A$ on $\mathbb{U}$ is called hyponormal if

$$
D(A) \subset D\left(A^{*}\right), \quad\left\|A^{*} u\right\| \leq\|A u\| \quad \text { for each } u \in D(A),
$$

where $D(A)$ and $D\left(A^{*}\right)$ denote the domains of $A$ and its adjoint $A^{*}$, respectively.

In view of the above, every (possibly not continuous) normal operator on $\mathbb{t}$ is hyponormal, the converse being obviously false. For details on hyponormal operators including a comprehensive investigation on their spectral properties, we refer the reader to $[7,8,9,16,17,18]$ and the references therein.

Let $A$ be a hyponormal operator on $\mathbb{W}$. One says that $A$ is of Putnam type if it can be expressed by

$$
A=K+i L
$$

where both $K, L$ are selfadjoint linear operators, $K$ is possibly unbounded, and $L$ is bounded on $\mathbb{H}$, see $[9,16]$, for details. ( $i$ being the complex number such that $i^{2}=-1$.) It should be noted that since $L$ is bounded, the adjoint $A^{*}$ of $A$ is defined by, $A^{*}=K-i L$, and hence $D(A)=D\left(A^{*}\right)=D(K)$.

We mention that the growing theory of hyponormal operators has been of great interest for several mathematicians in the past decades. Note the pioneer work of Putnam $[16,17]$, the recent contributions by Janas in $[7,8,9]$, and those of Xia in [18]. This paper goes back to revisit this class of operators, especially to find sufficient conditions such 
that if $A, B$ are closed hyponormal operators of Putnam type and upon some suitable assumptions, then for each $0<\alpha<1$,

$$
D\left((\overline{A+B})^{\alpha}\right)=D\left(A^{\alpha}\right) \cap D\left(B^{\alpha}\right)=D\left((\overline{A+B})^{* \alpha}\right)
$$

where $\overline{A+B}$ is the closure of the algebraic sum $A+B$ of $A$ and $B$.

As an application, we will consider a large class of Schrödinger's operators $-\Delta+Q$, where the (complex) potential $Q$ is chosen of the form $Q=Q_{1}+i Q_{2}$ with $Q_{1} \in L_{\text {loc }}^{1}\left(\mathbb{R}^{d}\right)$ and $Q_{2} \in L^{\infty}\left(\mathbb{R}^{d}\right)$. As in the abstract setting, it will be shown that under some additional assumptions,

$$
D\left((\overline{\Delta+Q})^{\alpha}\right)=\mathbb{M}^{2 \alpha}\left(\mathbb{R}^{d}\right) \cap D\left(Q_{1}^{\alpha}\right)=D\left((\overline{\Delta+Q})^{* \alpha}\right),
$$

for each $0<\alpha<1$, where $D\left(Q_{1}\right)$ denotes the domain of the multiplication operator related to the potential $Q_{1}$.

As a special case, with $\alpha=1 / 2$ in (1.3), we recover the well-known square root problem of Kato for the algebraic sum of hyponormal operators of Putnam type. Notice that similar investigations were made by the author regarding the square root problem of Kato for the algebraic sum of normal and $m$-sectorial operators, see, for example, $[3,4,5,6]$.

\section{Preliminaries}

Let $A, B$ be closed hyponormal linear operators acting on a (complex) Hilbert space $\mathbb{H}$. Recall that the algebraic sum $A+B$ of $A$ and $B$ is defined by

$$
\begin{gathered}
D(A+B)=D(A) \cap D(B), \\
(A+B) u:=A u+B u \quad \forall u \in D(A) \cap D(B) .
\end{gathered}
$$

For $0<\alpha<1$, let $A^{\alpha}$ and $B^{\alpha}$ denote the $\alpha$ th powers of $A$ and $B$, respectively (see [13, $14,15]$ for details). It should be noted that under assumptions (H.1), (H.3), and (H.4) below, it can be shown that the fractional powers $A^{\alpha}$ and $B^{\alpha}$ of $A$ and $B$ do exist.

For $0<\alpha<1$, the domains $D\left(A^{\alpha}\right)$ and $D\left(B^{\alpha}\right)$ of the fractional operators $A^{\alpha}$ and $B^{\alpha}$ are respectively defined through the following (complex) interpolation spaces:

$$
D\left(A^{\alpha}\right)=[D(A), \mathbb{H}]_{\alpha}, \quad D\left(B^{\alpha}\right)=[D(B), \mathbb{H}]_{\alpha} .
$$

Similarly, if the algebraic sum $A+B$ of $A$ and $B$ is nontrivial, then the domains of its fractional powers $(A+B)^{\alpha}$ are defined by

$$
D\left((A+B)^{\alpha}\right)=[D(A) \cap D(B), \mathbb{H}]_{\alpha} .
$$

Again, for details on fractional powers of operators, we refer the reader to $[11,12,13$, $14,15]$ and the references therein.

It should be observed that if $A, B$ are of Putnam type, that is,

$$
A=K+i L, \quad B=M+i R,
$$


where $K, L, M$, and $R$ are selfadjoint linear operators, $K, M$ are unbounded, and $L, R$ are bounded, then

$$
D(A)=D(|A|)=D(K), \quad D(B)=D(|B|)=D(M),
$$

where $|A|=\left(A^{*} A\right)^{1 / 2}$ and $|B|=\left(B^{*} B\right)^{1 / 2}$.

Consequently,

$$
\begin{aligned}
& D\left(A^{\alpha}\right)=D\left(|A|^{\alpha}\right)=D\left(K^{\alpha}\right), \\
& D\left(B^{\alpha}\right)=D\left(|B|^{\alpha}\right)=D\left(M^{\alpha}\right)
\end{aligned}
$$

for each $0<\alpha<1$.

The paper is organized as follows. In Section 3, it will be shown that under appropriate assumptions, the domain of the algebraic sum $A+B$ of $A$ and $B$ satisfies (1.3) (Theorem 3.1). In Section 4, the main result is applied to a large class of Schrödinger's operators with singular potential (Corollary 4.1).

Throughout the paper, $\mathbb{H}$, and $A$ and $B$ stand for a (complex) Hilbert space, closed (possibly unbounded) hyponormal linear operators acting into $\mathbb{Z}$, respectively. In addition to that we suppose that $A, B$ are of Putnam type, that is,

$$
A=K+i L, \quad B=M+i R,
$$

where $K, L, M, R$ are selfadjoint on $\mathbb{H}, K, M$ are unbounded, and $L, R$ are bounded linear operators.

For $0<\alpha \leq 1$, we set $V_{\alpha}=V\left(A^{\alpha}\right) \cap V\left(B^{\alpha}\right)$, where

$$
V\left(A^{\alpha}\right)=D\left(|A|^{\alpha}\right)=D\left(K^{\alpha}\right), \quad V\left(B^{\alpha}\right)=D\left(|B|^{\alpha}\right)=D\left(M^{\alpha}\right) .
$$

The following assumptions will be made:

(H.1) $L$ and $R$ are nonnegative;

(H.2) $\overline{D(K) \cap D(M)}=\mathbb{H}$;

(H.3) there exists $a>0:\langle L u, u\rangle \leq a\langle K u, u\rangle$, for each $u \in V\left(A^{1 / 2}\right)$;

(H.4) there exists $b>0:\langle R u, u\rangle \leq b\langle M u, u\rangle$, for each $u \in V\left(B^{1 / 2}\right)$.

Notice that from the assumptions (H.1) and (H.3), $A=K+i L$ is $m$-sectorial (see [12] for details). Similarly, the assumptions (H.1) and (H.4) yield that $B=M+i R$ is $m$ sectorial.

Under assumptions (H.1), (H.3), and (H.4), we consider the sesquilinear forms $\phi, \psi$ associated with $A, B$.

Consequently,

$$
\begin{aligned}
& \phi(u, v)=\left\langle K^{1 / 2} u, K^{1 / 2} v\right\rangle+i\left\langle L^{1 / 2} u, L^{1 / 2} v\right\rangle \quad \forall u, v \in V\left(A^{1 / 2}\right), \\
& \psi(u, v)=\left\langle M^{1 / 2} u, M^{1 / 2} v\right\rangle+i\left\langle R^{1 / 2} u, R^{1 / 2} v\right\rangle \quad \forall u, v \in V\left(B^{1 / 2}\right) .
\end{aligned}
$$

Next, let $\xi$ denote the sum of the sesquilinear forms $\phi$ and $\psi$. Thus $\xi$ can be expressed by

$$
\xi(u, v)=\phi(u, v)+\psi(u, v) \quad \forall u, v \in V_{1 / 2} .
$$


As in normal operators, see, for example, [2, page 451] or [10, pages 260-261], one can define the "generalized" sum or form sum, $A \dot{+} B$ of $A$ and $B$ with the help of the sesquilinear form $\xi$ as follows: $u \in D(A+B)$ if and only if the mapping, $v \rightarrow \xi(u, v)$ is continuous for the $\mathbb{U}$-topology and $(A \dot{+} B) \mathcal{u}$ defined to be the vector of $\mathbb{U}$ given by the Riesz representation theorem

$$
\langle(A \dot{+} B) u, v\rangle=\xi(u, v), \quad u \in D(A \dot{+} B) \quad \forall v \in V_{1 / 2} .
$$

Under assumption (H.2), it follows that $\xi$ has the following other representation:

$$
\xi(u, v)=\langle(A+B) u, v\rangle \quad \forall u \in D(A) \cap D(B), \forall v \in V_{1 / 2}
$$

\section{Fractional powers of operators}

In this section, it will be shown that the algebraic sum $S$ of $A$ and $B$ satisfies (1.1) under assumptions (H.1)-(H.4).

Theorem 3.1. Let $A=K+i L, B=M+i R$ be unbounded hyponormal operators of Putnam type on $\mathbb{H}$. Suppose that the assumptions (H.1)-(H.4) hold and that the operator $\overline{A+B}$ (closure of $A+B$ ) is maximal.

Then for each $0<\alpha<1$,

$$
D\left((\overline{A+B})^{\alpha}\right)=D\left(A^{\alpha}\right) \cap D\left(B^{\alpha}\right)=D\left((\overline{A+B})^{* \alpha}\right) .
$$

Proof. We first show that $\overline{A+B}$ is the operator associated with the sesquilinear form $\xi$ defined in (2.10). For that, consider the sesquilinear form $\xi=\phi+\psi$ in (2.10) and let $V_{\xi}=\left(V_{1 / 2},\langle\cdot, \cdot\rangle_{\xi}\right)$ be the pre-Hilbert space $V_{1 / 2}$ equipped with the inner product

$$
\langle u, v\rangle_{\xi}:=\langle u, v\rangle_{\mathbb{H}}+\mathfrak{R} e \xi(u, v) \quad \forall u, v \in V_{1 / 2} .
$$

Since the sum form $K \dot{+} M$ of $K$ and $M$ is a nonnegative selfadjoint operator, it easily follows that $V_{\xi}$ is a Hilbert space, hence $\xi$ is a closed sesquilinear form. (Assumptions (H.1), (H.3), and (H.4) yield that $K, M$ are nonnegative selfadjoint operators.) Moreover, since $D(\xi)=V_{1 / 2}$ is dense on $\mathbb{U}$, by $D(A) \cap D(B) \subset V_{1 / 2}$ and assumption (H.2), hence $\xi$ is a densely defined closed sesquilinear form.

From assumptions (H.3) and (H.4), it is clear that

$$
|\mathfrak{J} m \xi(u, u)| \leq C \cdot \mathfrak{R} e \xi(u, u) \quad \forall u \in V_{1 / 2},
$$

where $C>0$.

We then conclude that $\xi$ is a densely defined closed sectorial sesquilinear form. According to the first representation theorem [12, Theorem 2.1, page 322], there exists a unique $m$-sectorial operator associated with $\xi$, that is, $m$-sectorial extension of $A+B$. Now, $\overline{A+B}$ is maximal, it follows that $\overline{A+B}$ is the $m$-sectorial operator associated with $\xi$.

Now since $D(A)=D\left(A^{*}\right)=D(K)$ and $D(B)=D\left(B^{*}\right)=D(M)$, it follows that $D(\overline{A+B}) \subset D\left((\overline{A+B})^{*}\right)$, and therefore

$$
D\left((\overline{A+B})^{1 / 2}\right) \subset D(\xi)=V_{1 / 2} \subset D\left((\overline{A+B})^{* 1 / 2}\right) .
$$


Similarly from the inclusion $D(\overline{A+B}) \subset D((\overline{A+B}) *)$ and Kato [11], it follows that

$$
D\left((\overline{A+B})^{\alpha}\right) \subset V_{\alpha} \subset D\left((\overline{A+B})^{* \alpha}\right)
$$

for each $\alpha \in(0,1 / 2) \cup(1 / 2,1)$.

From (3.4) and (3.5), one obtains that

$$
D\left((\overline{A+B})^{\alpha}\right) \subset V_{\alpha} \subset D\left((\overline{A+B})^{* \alpha}\right)
$$

for each $\alpha \in(0,1)$.

Considering the conjugate $\xi^{*}$ of $\xi$ and using similar arguments as above for the adjoint $\overline{A+B}^{*}$ of $\overline{A+B}$, it follows that

$$
D\left((\overline{A+B})^{* \alpha}\right) \subset V_{\alpha} \subset D\left((\overline{A+B})^{\alpha}\right)
$$

for each $0<\alpha<1$.

One completes the proof using the fact that $D(\xi)=D\left(\xi^{*}\right)$ and combining (3.6) and (3.7) above, that is,

$$
D\left((\overline{A+B})^{\alpha}\right)=D\left(A^{\alpha}\right) \cap D\left(B^{\alpha}\right)=D\left((\overline{A+B})^{* \alpha}\right)
$$

for each $0<\alpha<1$.

\section{Applications}

This section provides an application to Theorem 3.1. Namely, we consider a large class of linear operators involving the well-known Schrödinger's operators, that is, $-\Delta+Q$, where $\Delta$ denotes the Laplace (differential) operator defined by

$$
\Delta:=\sum_{k=1}^{d} \frac{\partial^{2}}{\partial x_{k}^{2}}
$$

and the potential $Q$ belongs to $L_{\text {loc }}^{1}\left(\mathbb{R}^{d}\right)+L^{\infty}\left(\mathbb{R}^{d}\right)$.

Let $\mathbb{W}=L^{2}\left(\mathbb{R}^{d}\right)$ and let $\Phi$ be the sesquilinear form defined by

$$
\begin{gathered}
D(\Phi)=\mathbb{M}^{1}\left(\mathbb{R}^{d}\right), \\
\Phi(u, v)=\int_{\mathbb{R}^{d}} \nabla u \overline{\nabla v} d x \quad \forall u, v \in \mathbb{T}^{1}\left(\mathbb{R}^{d}\right) .
\end{gathered}
$$

Clearly, $\Phi$ is a sectorial sesquilinear form with domain $D(\Phi)=\mathbb{H}^{1}\left(\mathbb{R}^{d}\right)$.

On the other hand, suppose that the potential $Q$ can be written as

$$
Q=Q_{1}+i Q_{2},
$$

where $Q_{1}(x), Q_{2}(x) \geq 0$ (a.e). 
1930 Fractional powers of hyponormal operators

Let $\Psi$ be the sesquilinear form defined by

$$
\begin{gathered}
D(\Psi)=\left\{u \in L^{2}\left(\mathbb{R}^{d}\right): Q(x) \cdot|u(x)|^{2} \in L^{1}\left(\mathbb{R}^{d}\right)\right\}, \\
\Psi(u, v)=\int_{\mathbb{R}^{d}} Q u \bar{v} d x \quad \forall u, v \in D(\Psi) .
\end{gathered}
$$

Throughout this section we suppose $Q_{1} \in L_{\text {loc }}^{1}\left(\mathbb{R}^{d}\right), Q_{2} \in L^{\infty}\left(\mathbb{R}^{d}\right)$, and there exists $\theta \in$ $(0, \pi / 2)$ such that

$$
\int_{\mathbb{R}^{d}} Q_{2}(x) \cdot|u(x)|^{2} d x \leq \tan \theta \cdot \int_{\mathbb{R}^{d}} Q_{1}(x) \cdot|u(x)|^{2} d x
$$

for each $u \in D(\Psi)$.

Observe that from (4.5), it follows that $\Psi$ is a sectorial sesquilinear form. Actually,

$$
|\mathfrak{J} m \Psi(u, u)| \leq \tan \theta \cdot \mathfrak{R} e \Psi(u, u) \quad \forall u \in D(\Psi) .
$$

Under the above assumptions it is clear that $\Phi, \Psi$ are densely defined closed sectorial forms. Furthermore, it is not hard to see that the operators associated with $\Phi$ and $\Psi$ are respectively defined by

$$
\begin{gathered}
D(A)=\mathbb{M}^{2}\left(\mathbb{R}^{d}\right), \\
A u=-\Delta u \quad \forall u \in \mathbb{T}^{2}\left(\mathbb{R}^{d}\right), \\
D(B)=\left\{u \in L^{2}\left(\mathbb{R}^{d}\right): Q(x) u \in L^{2}\left(\mathbb{R}^{d}\right)\right\}, \\
B u=Q u \quad \forall u \in D(B) .
\end{gathered}
$$

It is also routine to check that both $A$ and $B$ are normal operators, and hence they are hyponormal operators. Clearly, $A, B$ are of Putnam type on $L^{2}\left(\mathbb{R}^{d}\right)$ since $A=K+$ $i L$, where $K=-\Delta$ and $L \equiv 0$ (selfadjoint linear operators with $K$ unbounded and $L$ bounded), and $B=M+i R$, where $M, R$ are selfadjoint operators with $M$ unbounded and $R$ bounded.

Observe that $D(A) \cap D(B) \supset C_{0}^{\infty}\left(\mathbb{R}^{d}\right)\left(C_{0}^{\infty}\left(\mathbb{R}^{d}\right)\right.$ being the space of functions of class $C^{\infty}$ with compact support on $\left.\mathbb{R}^{d}\right)$. In other words, $A+B$ is densely defined in $L^{2}\left(\mathbb{R}^{d}\right)$. Clearly, the assumptions (H.1)-(H.4) are achieved.

Now consider the sum of $\Phi$ and $\Psi$ above, that is, $\Xi=\Phi+\Psi$. Clearly, the operator associated with $\Xi=\Phi+\Psi$ is the closure of the algebraic sum $-\Delta+Q$. It is also well-known, according to Brézis and Kato [1] that the operator $(\overline{-\Delta+V})$ is $m$-sectorial. Actually, such an operator is explicitly defined by

$$
\begin{gathered}
D(\overline{-\Delta+Q})=\left\{u \in \mathbb{T}^{1}\left(\mathbb{R}^{d}\right): Q|u|^{2} \in L^{1}\left(\mathbb{R}^{d}\right), \Delta u+Q u \in L^{2}\left(\mathbb{R}^{d}\right)\right\}, \\
(\overline{-\Delta+Q}) u=-\Delta u+Q u
\end{gathered}
$$

for all $u \in D(\overline{-\Delta+Q})$. 
The previous discussion can be formulated as follows.

Corollary 4.1. Let $A=-\Delta$ and $B=Q$ be the operators defined above where the potential $Q=Q_{1}+i Q_{2}$ with $Q_{1} \in L_{\mathrm{loc}}^{1}\left(\mathbb{R}^{d}\right)$ and $Q_{2} \in L^{\infty}\left(\mathbb{R}^{d}\right)$. Suppose that $Q_{1}(x), Q_{2}(x) \geq 0$ (a.e) and that (4.5) holds, then

$$
D\left((\overline{\Delta+Q})^{\alpha}\right)=\mathbb{W}^{2 \alpha}\left(\mathbb{R}^{d}\right) \cap D\left(Q_{1}^{\alpha}\right)=D\left((\overline{\Delta+Q})^{* \alpha}\right)
$$

for each $0<\alpha<1$, where $D\left(Q_{1}\right)$ is the domain of the multiplication operator associated with the singular potential $Q_{1}$.

Proof. In view of the previous discussion, it is clear that both $A=-\Delta$ and $B=Q$ defined above, satisfy assumptions (H.1)-(H.4). One completes the proof by using Theorem 3.1 above.

\section{References}

[1] H. Brézis and T. Kato, Remarks on the Schrödinger operator with singular complex potentials, J. Math. Pures Appl. (9) 58 (1979), no. 2, 137-151.

[2] A. de Bivar-Weinholtz and M. L. Lapidus, Product formula for resolvents of normal operators and the modified Feynman integral, Proc. Amer. Math. Soc. 110 (1990), no. 2, 449-460.

[3] T. Diagana, Sommes d'opérateurs et conjecture de Kato-McIntosh [Sums of linear operators and the Kato-McIntosh conjecture], C. R. Acad. Sci. Paris Sér. I Math. 330 (2000), no. 6, 461-464 (French).

[4]_, Quelques remarques sur l'opérateur de Schrödinger avec un potential complexe singulier particulier [Some remarks on the Schrodinger operator with a particular singular complex potential], Bull. Belg. Math. Soc. Simon Stevin 9 (2002), no. 2, 293-298 (French).

[5] _ Variational sum and Kato's conjecture, J. Convex Anal. 9 (2002), no. 1, 291-294.

[6] Algebraic sum of unbounded normal operators and the square root problem of Kato, Rend. Sem. Mat. Univ. Padova 110 (2003), 269-275.

[7] J. Janas, On unbounded hyponormal operators, Ark. Mat. 27 (1989), no. 2, 273-281.

[8] _ On unbounded hyponormal operators. II, Integral Equations Operator Theory 15 (1992), no. 3, 470-478.

[9] - On unbounded hyponormal operators. III, Studia Math. 112 (1994), no. 1, 75-82.

[10] G. W. Johnson and M. L. Lapidus, The Feynman Integral and Feynman's Operational Calculus, Oxford Mathematical Monographs, Clarendon Press, Oxford University Press, New York, 2000.

[11] T. Kato, Fractional powers of dissipative operators. II, J. Math. Soc. Japan 14 (1962), 242-248.

[12] , Perturbation Theory for Linear Operators, Die Grundlehren der mathematischen Wissenschaften, vol. 132, Springer-Verlag, New York, 1966.

[13] J.-L. Lions, Espaces intermédiaires entre espaces hilbertiens et applications, Bull. Math. Soc. Sci. Math. Phys. R. P. Roumaine (N.S.) 2 (50) (1958), 419-432 (French).

[14] Espaces d'interpolation et domaines de puissances fractionnaires d'opérateurs, J. Math. Soc. Japan 14 (1962), 233-241 (French).

[15] A. Pazy, Semigroups of Linear Operators and Applications to Partial Differential Equations, Applied Mathematical Sciences, vol. 44, Springer-Verlag, New York, 1983.

[16] C. R. Putnam, The spectra of unbounded hyponormal operators, Proc. Amer. Math. Soc. 31 (1972), 458-464.

[17] Spectra of polar factors of hyponormal operators, Trans. Amer. Math. Soc. 188 (1974), $419-428$. 


\section{Fractional powers of hyponormal operators}

[18] D. Xia, Spectral Theory of Hyponormal Operators, Operator Theory: Advances and Applications, vol. 10, Birkhäuser Verlag, Basel, 1983.

Toka Diagana: Department of Mathematics, Howard University, 2441 Sixth Street NW, Washington, DC 20059, USA

E-mail address: tdiagana@howard.edu 


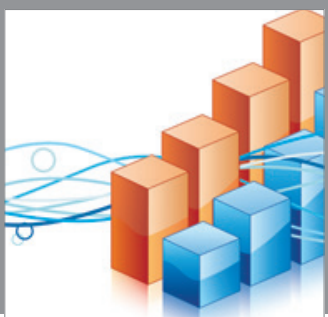

Advances in

Operations Research

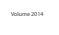

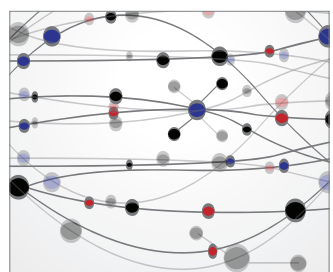

\section{The Scientific} World Journal
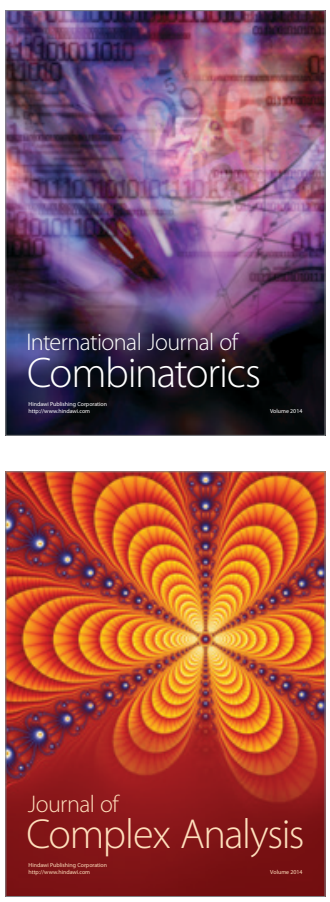

International Journal of

Mathematics and

Mathematical

Sciences
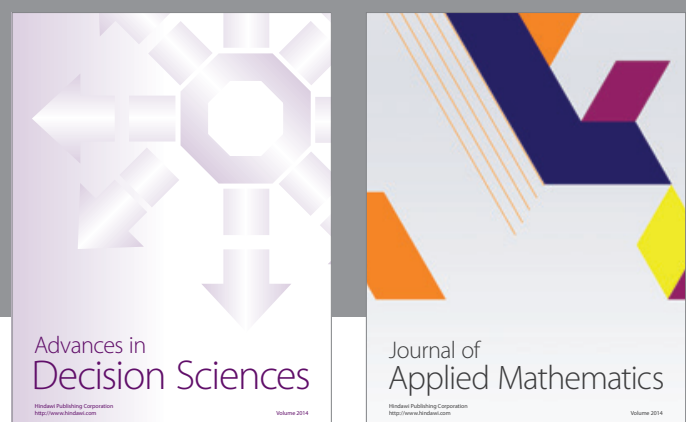

Journal of

Applied Mathematics
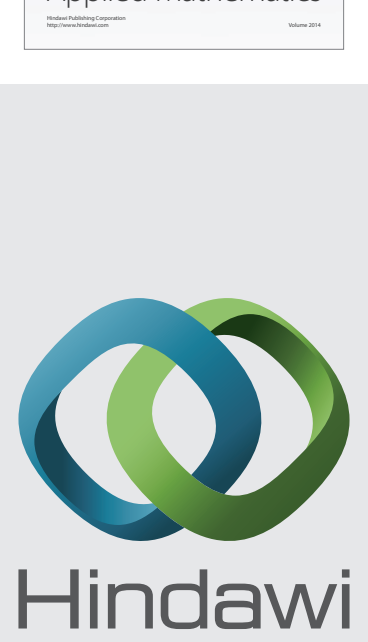

Submit your manuscripts at http://www.hindawi.com
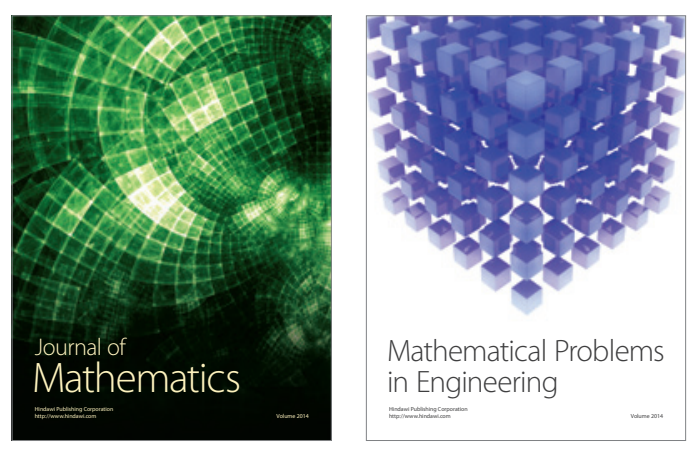

Mathematical Problems in Engineering
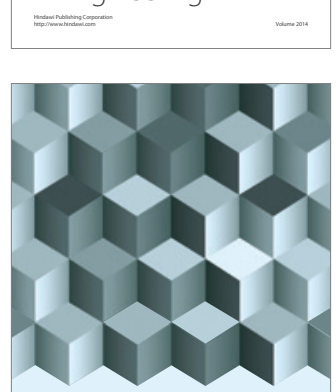

Journal of

Function Spaces
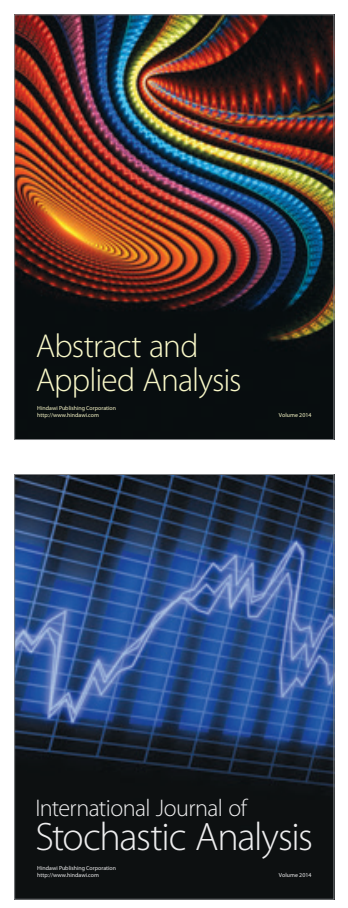

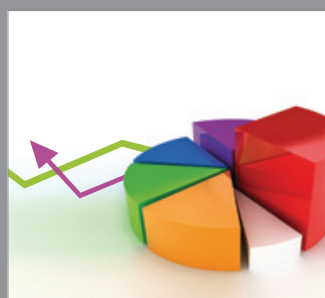

ournal of

Probability and Statistics

Promensencen
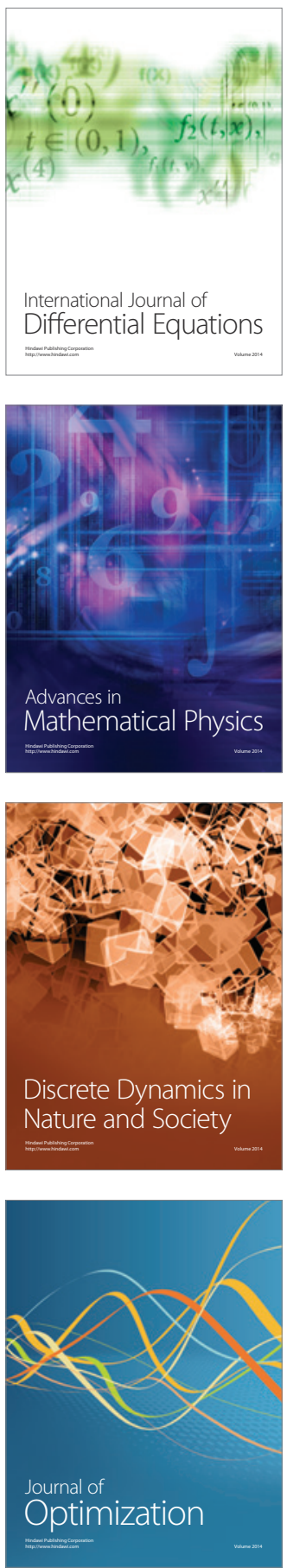